\title{
Cryoextraction of an Endobronchial Aspergilloma Through Rigid Bronchoscopy
}

\author{
Yang Zhou C, Aragaki-Nakahodo A, Luckett KM, Sen S and Radchenko C* \\ Pulmonology and Critical Care Medicine, Assistant Professor of Medicine, University of Cincinnati Medical Center, Cincinnati, USA
}

*Corresponding author: Christopher Radchenko, Pulmonology and Critical Care Medicine, Assistant Professor of Medicine,

University of Cincinnati Medical Center, Cincinnati, USA

\begin{abstract}
ARTICLE INFO
Received: 幽 October 29, 2019

Published: 幽 November 05, 2019

Citation: Yang Zhou C, Aragaki-Nakahodo A, Luckett KM, Sen S, Radchenko C. Cryoextraction of an Endobronchial Aspergilloma Through Rigid Bronchoscopy. Biomed J Sci \& Tech Res 22(3)-2019. BJSTR. MS.ID.003760.

ABSTRACT

Endobronchial aspergilloma is a rare entity that commonly affects the distal bronchial tree, often requiring surgical resection with concomitant systemic antifungal therapy. Diagnostic evaluation and therapeutic management are often fraught with inconsistent laboratory data, and challenging complications. We describe a unique case of invasive pulmonary aspergillosis presenting as an obstructing large airway endobronchial mass measuring $4.5 \mathrm{~cm}$ in largest dimension. Culture data and fungal biomarkers were negative for definitive infectious etiology. Utilizing a $1.9 \mathrm{~mm}$ cryoprobe through means of rigid bronchoscopy, the pulmonary aspergilloma was cryoextracted entirely without compromising its intrinsic structure. Given adverse effects from voriconazole, the patient was successfully treated with adjunctive isavuconazonium.
\end{abstract}

Keywords: Cryoextraction; Aspergilloma; Flexible Bronchoscopy; Rigid Bronchoscopy; Airway Obstruction; Endobronchial Mass; Isavuconazonium

\section{Introduction}

In the past few decades, flexible bronchoscopy has largely replaced rigid bronchoscopy as the tool of choice for diagnosis and management of endobronchial disease. However, there are still several indications for which rigid bronchoscopy excels, one of which is clearance of central airway obstruction. Here, we describe a unique method of complete extraction of a large endobronchial aspergilloma using a cryoprobe through rigid bronchoscopy.

\section{Case Description}

A 56-year-old male with a past medical history significant for heart failure with reduced ejection fraction, chronic kidney disease stage IV, uncontrolled type II diabetes mellitus, and coronary artery disease, presented with a month of increasing dyspnea on exertion afterahospitalizationforrecentlytreated pneumonia.Vitalsignswere notable for temperature 97.2 F, blood pressure 128/73 mmHg, heart rate 68 beats/minute, respiratory rate 16 breaths/minute, oxygen saturation $97 \%$ on 2 liters by nasal cannula. Physical examination was remarkable for a chronically ill appearing male, reduced breath sounds at the left base, dullness to percussion left posterior thorax, and a prior healed left below-knee amputation. Laboratory data revealed a white blood cell count of 9,500 cells $/ \mathrm{mm} 3$, hemoglobin of $9.6 \mathrm{gm} / \mathrm{dl}$, hematocrit $28.4 \%$, platelets of 159,000 cells $/ \mathrm{mm} 3$, glucose of $237 \mathrm{gm} / \mathrm{dl}$, blood urea nitrogen $53 \mathrm{mg} / \mathrm{dl}$, creatinine $4.5 \mathrm{mg} / \mathrm{dl}$. He underwent chest computed tomography, which was remarkable for a dense mass-like consolidation in the left lower lobe of the lung, with an endobronchial component, complicated by a moderate pleural effusion (Figure 1). He was started on vancomycin, piperacillin/tazobactam, and azithromycin for pneumonia with post-obstructive phenomenon and underwent inspection bronchoscopy. 


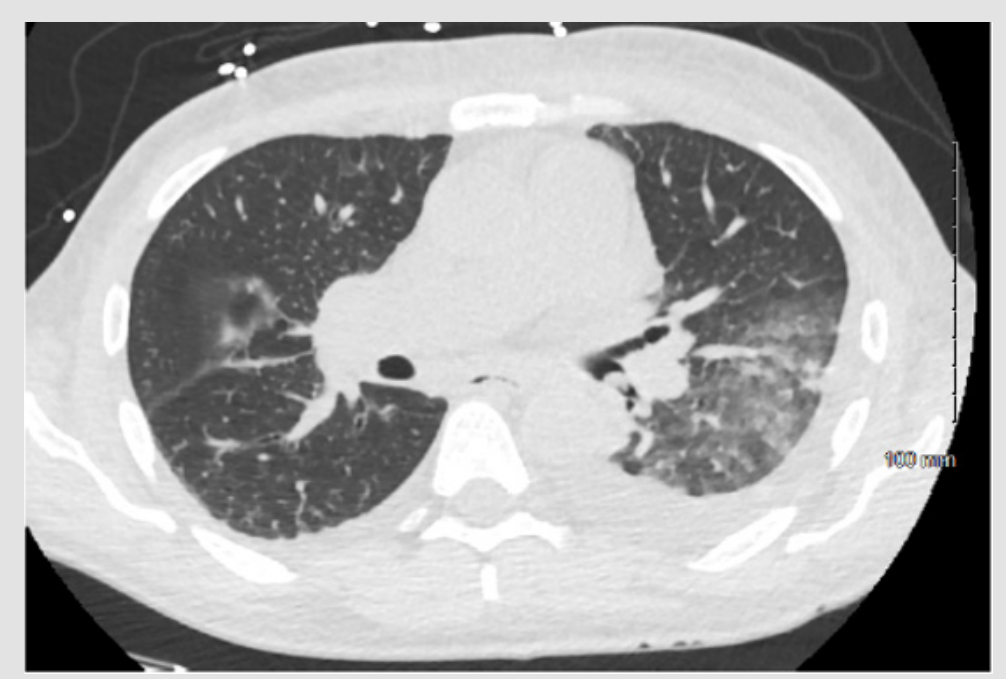

Figure 1: Chest computed tomography showing endobronchial lesion within the distal left mainstem bronchus, obstructing the left lower lobe. Further parenchymal opacities are seen in the left lower lobe, as well as a small to moderate left pleural effusion.

Inspection bronchoscopy was performed with a flexible bronchoscope, which revealed a distal left mainstem bronchial exophytic mass, which was causing near complete obstruction of the left lower lobe. An endobronchial forceps biopsy followed by brushing was completed, complicated by controllable bleeding, requiring application of topical cold saline. Bronchoalveolar lavage and brushings were both negative for identification of infection, however surgical pathology from endobronchial forceps of the mass showed fragmented fungal organisms. The patient was started on voriconazole $200 \mathrm{mg}$ PO q12h, and 1,3-beta-d-glucan and aspergillus biomarkers were drawn. Antifungal therapy was changed to isavuconazonium (372 mg PO q8h for 2 days, tapered to PO daily thereafter) given visual disturbances experienced with voriconazole. Aspergillus antigen index was negative at 0.03 , 1,3-beta-d-glucan was negative at $<34 \mathrm{pg} / \mathrm{ml}$, Aspergillus flavus and niger antibodies were not detected.

Rigid bronchoscopy was performed, utilizing full intravenous anesthesia with neuromuscular blockade, and automated jet ventilation with pressure-controlled settings. The trachea was intubated with a rigid 12.0-11.0 $\mathrm{mm}$ bronchoscope and advanced to the distal trachea. An endobronchial tumor was found at the entrance of the left lower lobe bronchus (Figure 2). The left mainstem bronchus was intubated and secured given concern of repeated bleeding. Careful inspection of the mass, as well as further characterization with a flexible bronchoscope caused immediate moderate bleeding of the surrounding mucosa, which subsided after endobronchial instillation of $0.3 \mathrm{mg}$ of topical epinephrine. A $1.9 \mathrm{~mm}$ cryoprobe was then used for cryorecannalization by contacting the central mass with the probe while continuously activating under direct visualization. Ensuring no adhered bronchial mucosa during freezing, after approximately 5-7 seconds activation, the mass was extracted en bloc through the rigid barrel. The mass measured $4.5 \mathrm{~cm}$ in larg- est dimension (Figure 3). Inspection beyond the prior obstruction of the segmental lower lobe airways revealed intense inflammation, post-obstructive purulence, with significant mucosal friability (Figure 4). The left lower lobe airways were therapeutically suctioned and a bronchoalveolar lavage was performed in the anteromedial basilar segmental bronchus. The patient had no immediate complications, including no significant post-extraction bleeding (Figure 5) and a post-procedure chest radiograph showed improved aeration of the left base. Aspergillus antigen index from the bronchoalveolar lavage was negative at 0.10 . Final pathology showed branching fungal hyphae with organisms invading the bronchial cartilage and fibrous tissue, consistent with an aspergilloma (Figure 6). The patient continued treatment with isavuconazonium for 12 weeks.

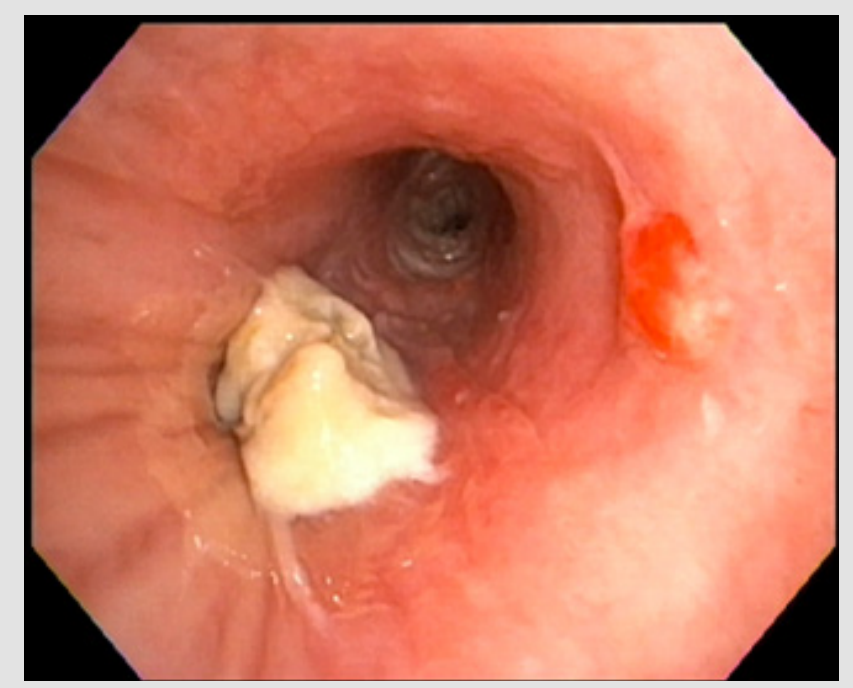

Figure 2: Bronchoscopic view of distal left mainstem bronchial exophytic lesion causing near complete obstruction of left lower lobe bronchi. 


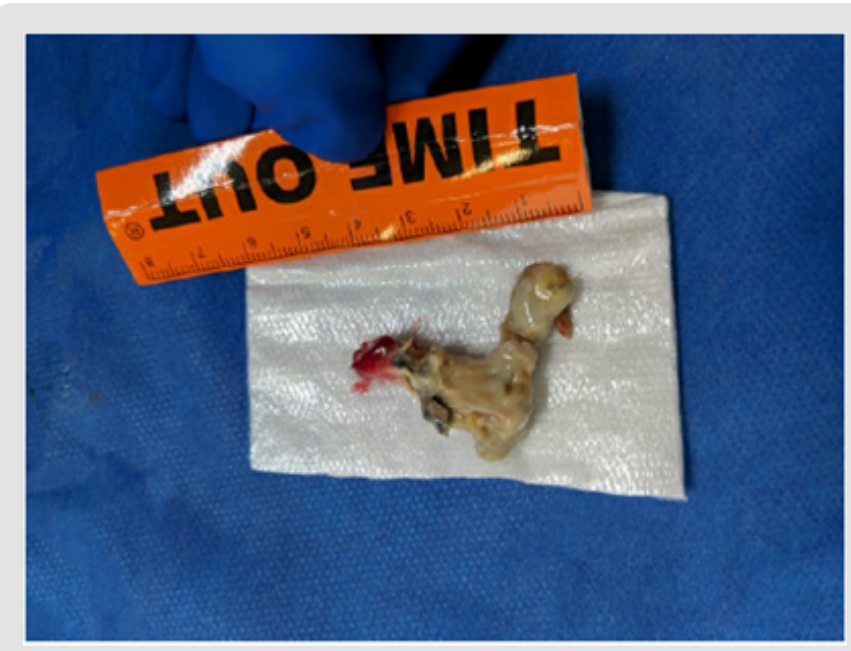

Figure 3: Pulmonary aspergilloma after cryoextraction en bloc through rigid bronchoscope. Maximal dimension 4.5 $\mathrm{cm}$ after removal with intrinsic structure entirely intact.

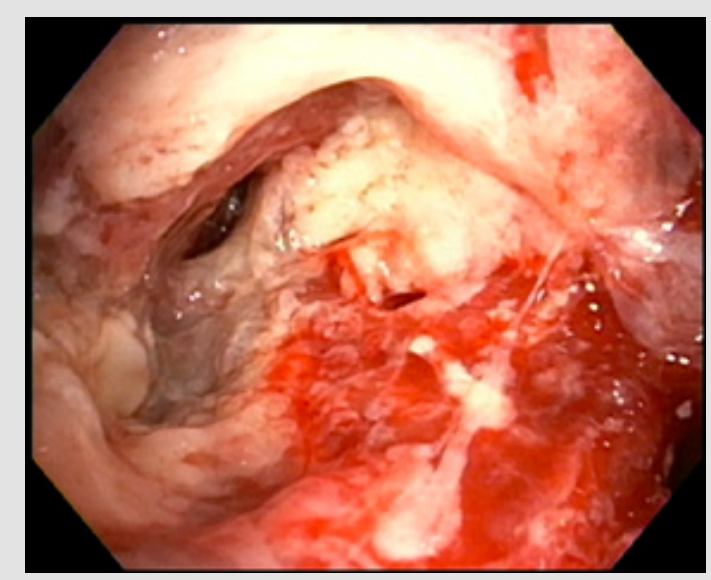

Figure 4: Bronchoscopic view of left lower lobe segmental bronchi post-cryoextraction of aspergilloma. Findings are notable for significant inflammation, post-obstructive purulence, and mucosal friability.

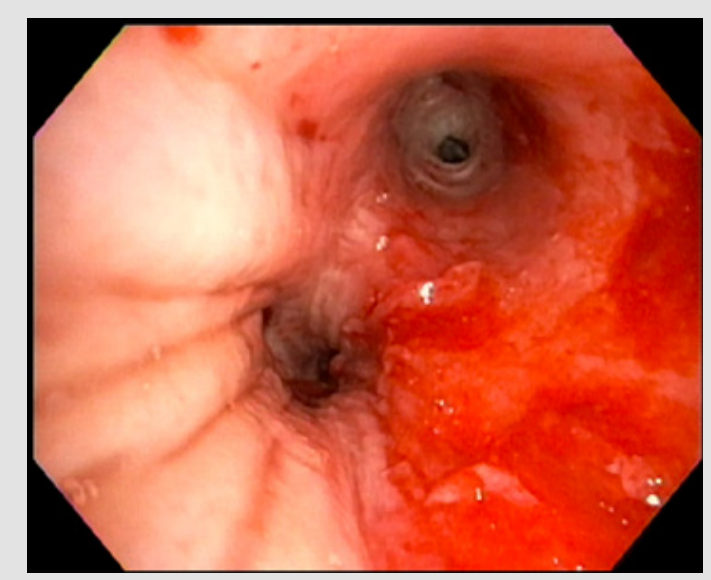

Figure 5: Bronchoscopic view of distal left mainstem bronchus leading to patent left lower lobe bronchi, postcryoextraction of pulmonary aspergilloma. Findings are notable for bleeding from irritated surrounding bronchial mucosa.

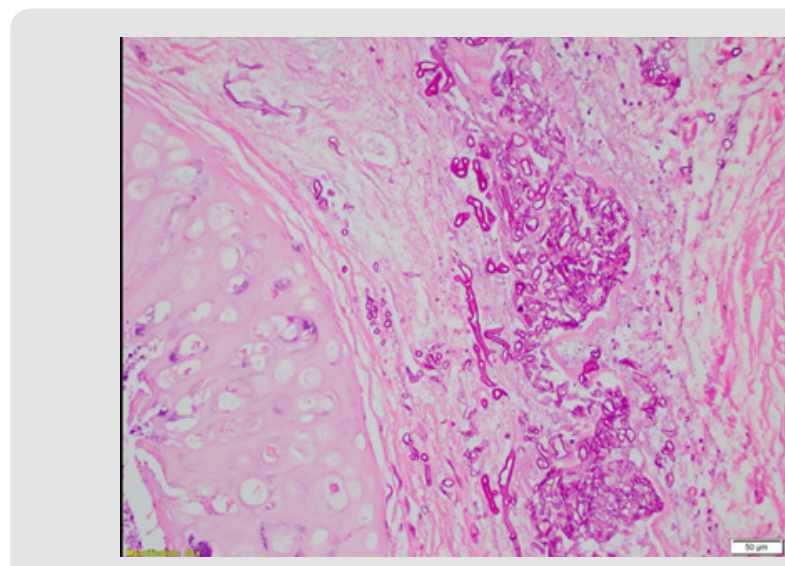

Figure 6: Surgical pathology specimen from extracted endobronchial mass. Hematoxylin and eosin staining are notable for branching filamentous hyphae consistent with Aspergillus spp.

\section{Discussion}

Aspergillus spp. is a ubiquitous organism that spreads through inhalation of spores. It has multiple presentations, including invasive and semi-invasive pulmonary aspergillosis, chronic necrotizing aspergillosis, and allergic bronchopulmonary aspergillosis. Endobronchial aspergilloma is an extremely rare presentation of infection with this organism with literature limited to case reports and short reviews summarizing them [1-4]. Our case described is unique in several ways. First, the aspergilloma presented in a more central airway, where all other cases have presented distally in the bronchial tree. Secondly, the aspergilloma was removed through rigid bronchoscopy with the use of a cryoprobe instead of traditional forceps. This technique allowed for entire extraction of the mass without disrupting its integral structure. To our knowledge, there have been no other published cases describing an endobronchial aspergilloma with either of these events. Finally, this case depicts the dilemma of confidently securing the diagnosis of an aspergilloma; although initial forceps was suggestive, culture data and fungal biomarkers were not, even in light of the size and invasion seen bronchoscopically.

All prior reports of endobronchial aspergilloma removal have been completed with a flexible bronchoscope alone [1]. While flexible bronchoscopes have largely replaced rigid bronchoscopes in practice [5], this case was well-suited for the use of rigid bronchoscopy due to the location of the mass within the bronchial tree, the size of the mass, as well as the risk of life-threatening bleeding with debulking. When removed, the mass measured $4.5 \mathrm{~cm}$ in maximal dimension. In a mass of this size, cryoextraction is often a more efficient approach for airway clearance as the cryoprobe often extracts a larger tissue sample than traditional forceps. Additionally, infectious masses often hold necrotic debris and are more cryosensitive due to increased water content, increasing efficacy of adhesion [5].

In cryorecanalization, a longer freezing time is used in hopes of removing large amounts of the mass at once. Rigid bronchoscopy 
can also assist here by "coring" out the mass with the beveled edge of the bronchoscope. The cryoprobe can then assist in removal of the mass in bulk. Here, rigid bronchoscopy can also provide immediate tamponade effect at the site of the mass, allowing for decreased bleeding, as well as enough space for additional suctioning even after removal of the flexible bronchoscope with the cryoprobe [6-7]. In a patient whose growth is likely to hold inflammatory or infectious material that can cause bronchial irritation, it may be improve patient recovery if the mass is removed in one piece without any spillage of its contents. As the working channel of flexible bronchoscopes is too small for the tissue samples obtained by cryoadhesion, the bronchoscope, cryoprobe, and tissue must be removed en bloc, thus requiring an advanced airway to ensure safe extraction. 6 However, if the internal diameter of the airway is also too small, the mass will be difficult to remove through the tube. Rigid bronchoscopy provides the largest diameter of the secured airway options [8]. There is limited data on the use of isavuconazonium for 12 weeks as adjunctive therapy for pulmonary aspergilloma. Treatment often involves pulmonary resection in conjunction with voriconazole being the typical antifungal of choice. However, there is no consensus on the utility of antifungal therapy after aspergilloma removal [9]. In immunocompetent patients, removal of the aspergilloma alone may be curative [10]. Further studies would be necessary to determine the appropriate antifungal agent and duration of therapy. In conclusion, the rarity of this disease presentation lends to a paucity of data to guide therapeutic decisions. Further research would be welcomed to determine the best treatment course for these pa- tients. For bronchoscopists, this case highlights the unique considerations in removal of infectious endobronchial masses as opposed the more common malignant or non-infectious benign growths.

\section{References}

1. Huang D, Li B, Chu H (2017) Endobronchial aspergilloma: A case report and literature review. Exp Ther Med 14(1): 547-554.

2. Sunnetcioglu A, Ekin S, Erten R, Parlak M, Esen R (2016) Endobronchial aspergilloma: A case report. Respir Med case reports 18: 1-3.

3. Ma JE, Yun EY, Kim YE (2011) Endobronchial Aspergilloma: Report of 10 Cases and Literature Review. Yonsei Med J 52(5): 787-792.

4. Jiang S, Jiang L, Shan F, Zhang X, Song M (2015) Two cases of endobronchial aspergilloma with lung cancer: a review the literature of endobronchial aspergilloma with underlying malignant lesions of the lung. Int J Clin Exp 8(9): 17015-17021.

5. DiBardino DM, Lanfranco AR, Haas AR (2016) Bronchoscopic Cryotherapy. Clinical Applications of the Cryoprobe, Cryospray, and Cryoadhesion. Ann Am Thorac Soc 13(8): 1405-1415.

6. Batra H, Yarmus L (2018) Indications and complications of rigid bronchoscopy. Expert Rev Respir Med 12(6): 509-520.

7. Schumann C, Hetzel M, Babiak AJ (2010) Endobronchial tumor debulking with a flexible cryoprobe for immediate treatment of malignant stenosis. J Thorac Cardiovasc Surg 139(4): 997-1000.

8. Shepherd RW, Radchenko C (2019) Bronchoscopic ablation techniques in the management of lung cancer. Ann Transl Med 7(15): 362.

9. Benhamed L, Woelffle D (2014) Adjuvant antifungal therapy after pulmonary surgery for aspergilloma: is it useful? Interact Cardiovasc Thorac Surg 18(6): 835-837.

10. Sagan D, Goździuk K (2010) Surgery for Pulmonary Aspergilloma in Immunocompetent Patients: No Benefit from Adjuvant Antifungal Pharmacotherapy. Ann Thorac Surg 89(5): 1603-1610.
ISSN: 2574-1241

DOI: 10.26717/BJSTR.2019.22.003760

Christopher Radchenko. Biomed J Sci \& Tech Res

This work is licensed under Creative Commons Attribution 4.0 License

Submission Link: https://biomedres.us/submit-manuscript.php

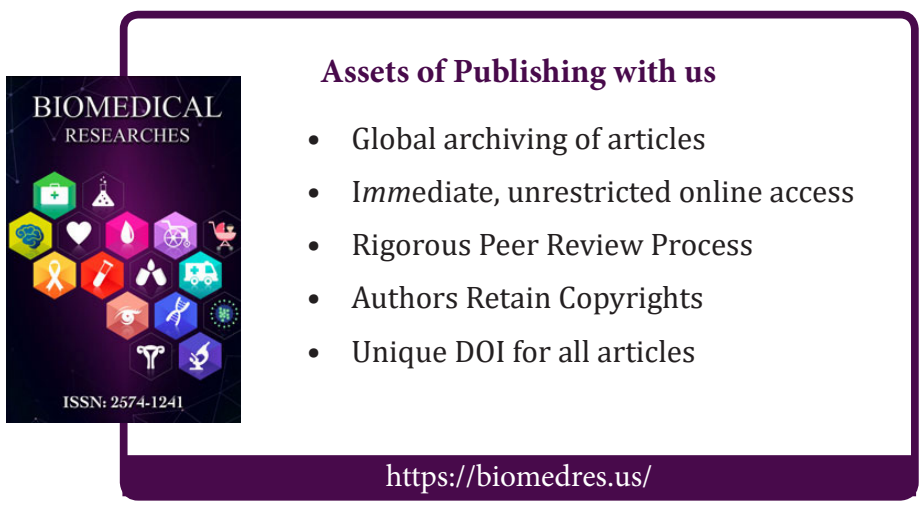

